\title{
Steroid-Induced Osteonecrosis of the Femoral Head: Novel Insight Into the Roles of Bone Endothelial Cells in Pathogenesis and Treatment
}

\author{
Cheng Huang ${ }^{1 \dagger}$, Zeqin Wen ${ }^{2 \dagger}$, Junjie $\mathrm{Niu}^{3 *}$, Subin $\mathrm{Lin}^{4 *}$ and Weiguo Wang ${ }^{1 *}$ \\ ${ }^{1}$ Department of Orthopedics, China-Japan Friendship Hospital, Beijing, China, ${ }^{2}$ Department of Orthopedics, Xiangya Hospital, \\ Central South University, Changsha, China, ${ }^{3}$ Department of Orthopedics, The First Affiliated Hospital of Soochow University, \\ Suzhou, China, ${ }^{4}$ Department of Orthopedics, The Second Affiliated Hospital of Soochow University, Suzhou, China
}

\section{OPEN ACCESS}

Edited by:

Phillip Trevor Newton,

Karolinska Institutet (KI), Sweden

Reviewed by:

Toshiki Kato,

University of Tsukuba, Japan

Marco Ponzetti,

University of L'Aquila, Italy

*Correspondence:

Junjie Niu

jjnewzzzx@sina.com

Subin Lin

tonylin23@126.com

Weiguo Wang

wangweiguo@zryhyy.com.cn

${ }^{+}$These authors have contributed equally to this work and share first authorship

Specialty section:

This article was submitted to Stem Cell Research,

a section of the journal

Frontiers in Cell and Developmental

Biology

Received: 15 September 2021 Accepted: 16 November 2021

Published: 30 November 2021

Citation:

Huang C, Wen Z, Niu J, Lin S and Wang W (2021) Steroid-Induced Osteonecrosis of the Femoral Head: Novel Insight Into the Roles of Bone Endothelial Cells in Pathogenesis and Treatment.

Front. Cell Dev. Biol. 9:7777697. doi: 10.3389/fcell.2021.777697
Steroid-induced osteonecrosis of the femoral head (SONFH) is a disease characterized by the collapse of the femoral head. SONFH occurs due to the overuse of glucocorticoids (GCs) in patients with immune-related diseases. Among various pathogenesis proposed, the mechanism related to impaired blood vessels is gradually becoming the most convincing hypothesis. Bone endothelial cells including bone microvascular endothelial cells (BMECs) and endothelial progenitor cells (EPCs) play a crucial role in the maintenance of vascular homeostasis. Therefore, bone endothelial cells are key regulators in the occurrence and progression of SONFH. Impaired angiogenesis, abnormal apoptosis, thrombosis and fat embolism caused by the dysfunctions of bone endothelial cells are considered to be the pathogenesis of SONFH. In addition, even with high disability rates, SONFH lacks effective therapeutic approach. Icariin (ICA, a flavonoid extracted from Epimedii Herba), pravastatin, and VO-OHpic (a potent inhibitor of PTEN) are candidate reagents to prevent and treat SONFH through improving above pathological processes. However, these reagents are still in the preclinical stage and will not be widely used temporarily. In this case, bone tissue engineering represented by co-transplantation of bone endothelial cells and bone marrow mesenchymal stem cells (BMSCs) may be another feasible therapeutic strategy.

Keywords: steroid-induced osteonecrosis of the femoral head, bone endothelial cells, bone microvascular endothelial cells, bone progenitor cells, angiogenesis, pathogenesis, treatment

\section{INTRODUCTION}

Glucocorticoids have been widely used in the treatment of rheumatic diseases, autoimmune diseases and allergic diseases (Yao et al., 2020). However, long-term or extensive GCs use may lead to steroidinduced osteonecrosis of the femoral head (SONFH). SONFH is a disabling orthopedic disease, which is characterized by the progressive deterioration of the hip joint in individuals aged 20-50 years old (Kong et al., 2020; Yu et al., 2020). Although many pathophysiological mechanisms for SONFH have been proposed, such as impaired microcirculation, imbalance between osteogenic and adipogenic differentiation, fat embolism, coagulation disorder and intramedullary pressure change, significant gaps remain in the understanding of the 
pathogenesis of SONFH (Li et al., 2005; Murata et al., 2007; Yeh et al., 2008; Yue et al., 2021). Among several existing mechanisms for SONFH, the vascular hypothesis seems to be the most convincing and influential (Kerachian et al., 2006).

As long ago as 1935, Phemister raised that vascular impairment led to thrombosis and embolism contributing to the progression of avascular necrosis of the femoral head (ANFH) (Phemister, 1935). It was not until Hirano et al. that direct histological evidence, severe luminal stenosis of the draining vein in the early stages of ANFH, was observed (Hirano et al., 1997). In another study, Starklint et al. have found a wide obstruction of vessels in the late stages of ANFH and the venous outflow is further damaged by thrombus and perivenous concentric fibrosis, which immensely reduces the lumen of veins (Starklint et al., 1995). In addition, osteocytes cannot survive more than $100 \mathrm{~mm}$ from blood vessels, so it is widely believed that vascular development always precedes osteogenesis ( $\mathrm{Yu}$ et al., 2009). However, with the deepening understanding of bone formation and repair, "angiogenicosteogenic coupling" concept has been established (Riddle et al., 2009). SONFH is a type of ANFH. As a result, blood vessels play a key role in the pathogenesis and repair of SONFH. Currently, vascular hypothesis assumes that GCs could reduce the number of blood vessels, decrease the regional blood flow of femoral head and lead to SONFH (Kerachian et al., 2006; Kerachian et al., 2009a).

Bone endothelial cells mainly refer to bone microvascular endothelial cells (BMECs) and endothelial progenitor cells (EPCs) that can differentiate into BMECs. BMECs line the sinusoids and inner layer of blood vessels, which play a crucial role in vascular homeostasis and angiogenesis (Kusumbe et al., 2014). It is reported that femoral head microcirculation disorder secondary to BMECs dysfunction is of great significance in the occurrence and progression of SONFH (Nishimura et al., 1997; Kerachian et al., 2009b). Similarly, as the precursor cells of BMECs, EPCs are involved in maintaining the physiological structure and function of vascular endothelium (Yao et al., 2020). Several studies have shown that the number and function of circulating EPCs in patients with SONFH are impaired (Feng et al., 2010; Chen et al., 2013; Ding P. et al., 2019). Given to the importance of vascular hypothesis in SONFH, researches about the effects of BMECs and EPCs on the blood supply of femoral head are helpful to further understand the pathogenesis of SONFH.

Moreover, drug treatment (e.g., anticoagulants, fibrinolysisenhancing drugs, blood vessel dilatators and lipid-reducing drugs) combined with hip-preserving surgery (e.g., core decompression, bone transplantation and osteotomy) can be applied to treat early ONFH (Zhao et al., 2020). However, these treatments are less effective, as more than $80 \%$ of patients with ONFH eventually require total hip arthroplasty (THA) (Johnson et al., 2014). Although THA significantly improves the living quality of patients, it cannot be considered as the best therapy for ONFH because of dislocation, periprosthetic fracture, infection and prosthesis loosening after THA, especially in relatively young patients (Xu et al., 2021). Therapeutic strategies designed according to the pathophysiological role of BMECs and EPCs in SONFH pathogenesis may be effective.

In this review, we summarize the novel roles of bone endothelial cells in the pathogenesis and treatment of SONFH.
Impaired angiogenesis, abnormal apoptosis, thrombosis and fat embolism caused by the dysfunctions of bone endothelial cells are considered to be the pathogenesis of SONFH. Targeting to repair the amount and function of bone endothelial cells or cotransplantation of bone endothelial cells and bone marrow mesenchymal stem cells (BMSCs) may be effective therapeutic approaches with great application potential. Furthermore, it is promising to point out the direction of future studies on the pathogenesis and treatment of SONFH.

\section{BONE MICROVASCULAR ENDOTHELIAL CELLS}

As previously mentioned, BMECs line the interior surface of bone microvessels and sinuses, maintaining local blood supply in femoral head. Besides, the reduction of blood flow in femoral head plays a vital role in the pathogenesis of ANFH (Kerachian et al., 2006). Therefore, BMECs damage may be the critical factor to promote the progression of SONFH.

Recent studies have demonstrated the existence of two types of BMECs: type $\mathrm{H}$ and type $\mathrm{L}$ endothelial cells (Kusumbe et al., 2014). Type H BMECs are mainly located in metaphysis and highly express CD31 and endomucin (EMCN), while type L BMECs are mainly located in the diaphysis and lowly express CD31 and EMCN (Zhang J. et al., 2020). Runx2 ${ }^{+}$ osteoprogenitors and collagen type $1 \alpha^{+}$osteoblasts are abundant around the type $\mathrm{H}$ BMECs in the metaphysis and endosteum, suggesting type $\mathrm{H}$ BMECs could promote bone repair and regeneration (Kusumbe et al., 2014). However, there is almost no osteoprogenitor surrounding type L BMECs (Kusumbe et al., 2014; Xu et al., 2018). At present, there are few studies on the role of type H BMECs in the pathogenesis of SONFH. Some studies have even shown contradictory results, which may be attributed to the differences in preclinical animal models (Zhou et al., 2017; Lane et al., 2018; Peng et al., 2020). And whether targeting type $\mathrm{H}$ BMECs can reverse the pathological processes of SONFH remains unclear. Hence this review mainly focuses on recent research progresses of BMECs in SONFH.

\section{Animal Experiments of Bone Microvascular Endothelial Cells}

Patients with SONFH have common pathological features of allergic vasculitis prior to hormone administration (Saito et al., 1992). Lipopolysaccharide (LPS) stimulates the immune system and induces the pathological changes of the blood system. Therefore, the combination of LPS and methylprednisolone (MPS) to induce SONFH in Sprague-Dawley (SD) rats is consistent with human clinical pathological features (Saito et al., 1992). At the same time, femoral tissues of SD rats are collected for pathological examination to determine whether SONFH models are successfully established (Drescher et al., 2011). And the BMECs used in vitro were isolated from the femoral head of SONFH rat models. Animal experiments including in vivo and in vitro SONFH models were established using the above methods. 
So far, mechanisms regarding how the glucocorticoid takes effect on BMECs in animal experiments mainly focused on MicroRNAs (miRNAs). miRNAs are a group of small 18-25nt-long non-coding RNAs (Krol et al., 2010). They are involved in plenty of physiological and pathological processes by modulating the transcription or post-transcriptional translation to silence the expression of their target genes (Ambros, 2004; Lan et al., 2015). Four miRNAs differentially expressed in BMECs of SONFH rats were identified by real-time quantitative polymerase chain reaction (qPCR) and gene microarray, including two upregulated (miR-335, miR-132-3p) and two down-regulated (miR-466b-2-3p, let-7c-1-3p) (Yue et al., 2018). Moreover, Yue et al. reported that miR-335 could down-regulate the expression of endothelial nitric oxide synthase (eNOS), superoxide dismutase 2 (SOD2) and Ras p21 protein activator 1 (RASA1) (Yue et al., 2018). eNOS is a specific protease in BMECs, which has a variety of physiological effects, such as vasodilation, antiplatelet aggregation, and promoting functional repair of impaired BMECs (Yue et al., 2018). SOD is an antioxidant enzyme that catalyzes the reactive oxygen species (ROS) into hydrogen peroxide and oxygen molecules to inhibit senescence and apoptosis (Nguyen et al., 2020). RASA1 is a modulator of Ras GDP and GTP and plays an important role in several physiological processes such as angiogenesis, cell proliferation and apoptosis (Zhang Y. et al., 2020). In addition, Lei et al. observed that miR-132-3p expression was significantly upregulated after femoral artery occlusion, and the hind limb perfusion recovery after ischemia was slower in knockout mice compared with wild-type mice (Lei et al., 2015; Yue et al., 2018). Therefore, miR-335 and miR-132-3p may be involved in regulating the functional repair of impaired BMECs and angiogenesis in SONFH. However, the effects of rno-let-7c-1$3 \mathrm{p}$ and rno-miR-466b2-3p on proliferation and apoptosis of BMECs have not been reported (Yue et al., 2018). In the meantime, no studies have evaluated the role of glucocorticoid receptor (GR) on BMECs in the pathogenesis of SONFH. Whereas, a recent study investigated GR on mouse endothelial cells, identifying the pivotal role of Wnt signaling pathway in suppressing vascular inflammation via GR (Zhou et al., 2020). This result may guide the further research of signaling pathways mediated by GR on BMECs, which function as key factors in SONFH pathogenesis.

As a flavonoid extracted from Epimedii Herba, Icariin (ICA) has been widely used to promote bone healing, improve osteoporosis and SONFH in China, Japan and Korea (Brandi and Collin-Osdoby, 2006; Zhang et al., 2007; Zhu et al., 2012; Sun et al., 2015). Yue et al. (2021) observed that though still higher than the control group, the expression of miR-335 was markedly decreased after ICA treatment in vivo. What's more, they also found ICA had a modulatory effect on 101 unconventionally expressed target genes of miR-335 (Yue et al., 2021). As a result, down-regulating the expression of miR-335 may be the mechanism of ICA to prevent and therapy SONFH. In addition, Wen et al. (2008a) observed the increased ratio of empty lacunae, the sparse capillary network, and the partially blocked blood vessels in necrotic femoral head tissue from SONFH rabbits. However, ICA treatment can significantly decrease the rate of empty lacunae and relatively up-regulate the expression of angiogenic biomarker CD31 in vivo (Yu et al., 2019). And the tube formation and osteogenesis-related cytokines expression of BMECs can be stimulated by ICA in vitro (Yu et al., 2019). These results both in vitro and in vivo suggest that ICA may be a potential drug in the treatment of SONFH. However, rat models are far too different from human beings to infer similar therapeutic roles in humans.

\section{Human Experiments of Bone Microvascular Endothelial Cells}

There are two ways to establish the BMECs model with SONFH used in human experiments (Lu et al., 2020; Yu et al., 2020). One is to isolate BMECs from patients with SONFH and indications for THA, the other is to extract BMECs from patients with femoral neck fractures who have undergone THA. Yu et al. (2020) demonstrated that BMECs from SONFH patients had down-regulated angiogenic abilities. Endothelial function has been reported to decline with an increasing age in healthy individuals (Yavuz et al., 2008). However, dysfunction of BMECs was observed even when the mean age of the control group was significantly older than that of the SONFH group (Yu et al., 2020). This fully confirms that GCs can promote the progression of the dysfunction of BMECs from SONFH patients. However, the research results might not be replicated in the local microenvironment of the femoral head in the presence of SONFH because the study was conducted in vitro (Yu et al., 2020).

Similarly, Yu et al. (2019) also reported that hydrocortisone significantly inhibited the expression of angiogenic cytokines and the activation of Akt in BMECs, which decreased the migration and tube formation activities of BMECs. Angiogenic cytokines including vascular endothelial growth factor (VEGF), CD31, von Willebrand factor (vWF) and platelet-derived growth factor-B (PDGF-B) are promotors or markers of angiogenesis mainly expressed in BMECs (Yang et al., 2003; Muraoka et al., 2005; Uras et al., 2012; Mittermayr et al., 2016). It has been reported that the activation of survival signal PI3K/Akt pathway is related to angiogenesis (Lee et al., 2014). Since blood supply is critical to the maintenance of femoral head structure and function, dysfunction of BMECs and inhibited angiogenesis are potential mechanisms for SONFH (Kerachian et al., 2006).

Besides, some studies have found that GCs-induced apoptosis of BMECs can activate thrombosis and decrease angiogenesis, secondary by infarction and ischemia (Vogt and SchmidSchönbein, 2001; Kerachian et al., 2006). B cell lymphoma-2 (Bcl-2), as an oncoprotein, has a significant effect on inhibiting apoptosis, while Bcl-2 associated X (Bax) has an obvious effect on promoting apoptosis (Nomura et al., 1999; Delbridge et al., 2016). Therefore, it's the balance between Bcl-2 and Bax that determines apoptosis. Furthermore, caspase- 3 is a key factor in the activation of apoptosis (Porter and Jänicke, 1999). Yu et al. found the expression of Bcl-2 was significantly down-regulated, while the expression of Bax and cleaved caspase-3 were increased in BMECs with SONFH (Yu et al., 2019; Yu et al., 2020). These results demonstrate that the apoptosis of BMECs functions a lot in the progression of SONFH. 
In addition to impaired angiogenesis and increased apoptosis of BMECs, Li et al. (2004) reported that the hypercoagulability and hypofibrinolysis state induced by dysfunction of BMECs may be the pathological mechanism of SONFH as well. eNOS and endothelin 1 (ET-1) are two vasoactive factors with opposite functions secreted by BMECs, whose balance plays an important role in regulating vasomotor (Lu et al., 2020). ET-1 is the strongest vasoconstrictor until now and acts by binding to receptors on BMECs and vascular smooth muscle cells, while eNOS is a vasodilator and anticoagulation that acts by inhibiting the secretion and function of ET-1, platelet aggregation and intercellular adhesion (Houde et al., 2016; Hong et al., 2019). Angiotensin II (Ang II) binds to receptors on BMECs to inhibit eNOS expression and damage BMECs (Shatanawi et al., 2015). Prostaglandin $\mathrm{I}_{2}\left(\mathrm{PGI}_{2}\right)$ is secreted by BMECs and significantly expands blood vessels and suppresses platelet aggregation by activating prostacyclin receptors (IP receptors) in BMECs and platelets (Shatanawi et al., 2015). Prostaglandin $\mathrm{E}$ (PGE) is capable of expanding blood vessels, protecting BMECs and increasing the expression of eNOS (Fang et al., 2010). Plasminogen activator inhibitor-1 (PAI-1) is the inhibitor of tissue plasminogen activator ( $t-P A$ ) primarily produced by BMECs, the increased expression of which can promote intravascular thrombosis (Ghosh and Vaughan, 2012). Intercellular adhesion molecule 1 (ICAM-1), an important adhesion molecule, mediates adhesion between leukocytes, inflammatory cells and BMECs (Bui et al., 2020). Lu et al. found that the expressions of ET-1 receptor, Ang II receptor and ICAM-1 were dramatically increased and the expressions of eNOS, $\mathrm{PGI}_{2}$ synthase, PGE synthase, PGE receptor and VEGF were dramatically decreased after 24-h GCs treatment (Lu et al., 2020). However, the expression of ET-1 was dramatically down-regulated, suggesting that the effect of GCs on BMECs is complex and needs further investigations. In other words, vasoconstriction and thrombosis were promoted after GCs-induced BMECs damage.

Yu et al. (2019) reported that ICA could promote angiogenesis by up-regulating the expression of CD31, vWF, PDGF-B in BMECs and activating Akt and reduce the apoptosis of BMECs by up-regulating $B a x$ and down-regulating the expression of Bcl-2. Circular RNAs (circRNAs), serve as endogenous RNAs competing for miRNA binding sites, are regarded as new modulators of diseases (Wu et al., 2019). Mao et al. (2021) reported that CircCDRlas, functioning as a sponge for miR-135b/factor inhibiting hypoxia inducible factor 1 (FIH1 ), reduced the expression of hypoxia inducible factor-1a (HIF$1 \alpha)$ and VEGF, and thereby inhibited the angiogenesis of BMECs. Research results above suggest that the administration of ICA or targeting to inhibit CircCDR1 as may be effective therapeutic strategies for SONFH. However, the therapeutic approaches are still in the pre-clinical stage and lack the support of clinical trials. In addition, there is a short of therapeutic strategies targeting thrombosis caused by BMECs damage. Therefore, further investigations are needed in the future in regard to the thrombosis of SONFH.

\section{ENDOTHELIAL PROGENITOR CELLS}

EPCs are considered to be critical participants in endogenous vascular repair and regeneration by differentiating into mature endothelial cells (Kim et al., 2010; Balistreri et al., 2015). EPCs are primarily derived from bone marrow (Asahara et al., 2011). In addition, a small amount of EPCs are also found in umbilical cord blood, circulating blood and arterial walls (Doyle and Caplice, 2005; Wu et al., 2005; Finney et al., 2006; Pacilli and Pasquinelli, 2009). According to the difference in culture time, EPCs can be divided into two subgroups: early EPCs (eEPCs) and late EPCs (lEPCs) (Patel et al., 2016). In terms of maturation time, eEPCs appeared 4-7 days after culture, while lEPCs appeared 14-21 days after culture (Yang et al., 2018). eEPCs are characterized by several surface markers of progenitor cells, including CD14, CD31, CD34, CD45, CD133 and vWF (Recchioni et al., 2016). eEPCs have a weak proliferation capacity, but can secrete a variety of cytokines, such as VEGF, hepatocyte growth factor (HGF), granulocyte colony-stimulating factor (G-CSF), and interleukin-8 (IL-8) (Rehman et al., 2003). However, IEPCs express endothelial markers such as KDR, VEcadherin and CD146 with a strong capacity of proliferation and differentiation (Hirschi et al., 2008; Madonna and De Caterina, 2015). In fact, the antigen expression profile on the surface of EPCs remains controversial (Werner and Nickenig, 2006; Chen et al., 2013). When different combinations of surface antigens are selected, there may be some differences in experimental results (Ding P. et al., 2019).

EPCs have the potential to differentiate into any kinds of capillary endothelial cells, including BMECs (Peters, 2018). In addition, EPCs can be isolated noninvasively from the donors' own peripheral blood and umbilical cord blood, as well as from human induced pluripotent stem cells (hiPSCs) to avoid immunogenicity problems (Boyer et al., 2000; Ingram et al., 2004; Mead et al., 2008). EPCs-differentiated endothelial in vitro and in vivo have similar permeability to vessel-derived endothelial, and are superior to vessel-derived endothelial in vascular network formation (Peters, 2018). Therefore, EPCs transplantation to promote angiogenesis at the lesion region has great prospects. One of the most important pathogenesis of SONFH is the suppression of angiogenesis caused by dysfunction of BMECs, so most of the previous studies on SONFH focused on the changes of BMECs. However, recent studies have found that EPCs are more involved in vascular repair and regeneration than BMECs, which makes EPCs the focus of interest in the pathogenesis and treatment of SONFH (Ding S. et al., 2019).

\section{Animal Experiments of Endothelial Progenitor Cells}

Animal models for EPCs-related experiments were established by rats or rabbits treated with LPS and MPS/dexamethasone (Dex). Reduced blood flow and impaired blood supply to the femoral head caused by SONFH can lead to increased lactic acid levels resulting in an acidic local microenvironment (Song et al., 2010). Ovarian cancer G-protein-coupled Receptor 1 (OGR1) is a key 
receptor involved in sensing proton. Ding S. et al. (2019) found that OGR1 inhibited the proliferation, migration and angiogenesis of EPCs induced by acidic environment in SONFH. It means OGR1 may be a new breakthrough in treating SONFH. Moreover, it is well-known that stromal cellderived factor-1a (SDF-1a), the product of CXCL12, promotes angiogenesis through the CXCL12/CXCR4 or CXCL12/CXCR7 signaling pathway (Dimova et al., 2019; Zhang et al., 2019). Kong et al. (2020) demonstrated that transplantation of miR-137-3psilenced BMSCs can promote angiogenesis by up-regulating CXCL12/SDF-1 $\alpha$ to mobilize EPCs into circulation. However, whether CXCL12/CXCR4 or CXCL12/CXCR7 signaling pathways is involved in the mobilization of EPCs remains unknown.

In addition to the impaired angiogenesis caused by the damage of EPCs, the apoptosis of EPCs is also one of the possible pathogenesises of SONFH. Liao et al. (2017) reported that suppressed mammalian target of rapamycin (mTOR) signal induced by the activations of glucocorticoid receptors downregulates the HIF pathway and induces EPCs apoptosis, which may be the pathophysiological mechanism of SONFH. Meanwhile, there may be certain therapeutic potential in enhancing mTOR signal. Autophagy is a complex process in which cells adapt to degrade and recycle intracellular components under stress conditions, thus promoting cell survival (HamacherBrady et al., 2006; Eisenberg-Lerner et al., 2009). It was observed that autophagy increased in EPCs treated with Dex, but this change gradually attenuated with the prolongation of Dex treatment (Liao et al., 2018). At the same time, prolonged Dex treatment reduced cell viability, indicating that autophagy is beneficial for EPCs to respond to Dex stimulation and avoid damage (Liao et al., 2018). Liao et al. also reported that pravastatin activated AMP-activated protein kinase (AMPK) mediated by liver kinase B1 (LKB1), thereby inhibiting the mTOR signaling pathway, recovering autophagy of EPCs and protecting them from Dex-induced apoptosis (Liao et al., 2018). The above studies on mTOR signaling pathway have produced opposite conclusions, so the mechanism of mTOR signaling pathway in apoptosis of EPCs remains to be explored.

The extrinsic death receptor pathway and the intrinsic mitochondrial pathway are two main systems that initiate apoptosis (Thorburn, 2004). Phosphatase and tensin homolog (PTEN), a tumor-suppressor gene that enhances apoptosis, has recently been observed to be significantly elevated in the serum of patients with SONFH (Kotelevets et al., 2018; Li et al., 2018; Li et al., 2019). Moreover, Yao et al. (2020) found that GCs can induce EPCs apoptosis by activating mitochondrial pathway. VO-OHpic, a potent inhibitor of PTEN, could protect EPCs from apoptosis through inhibiting mitochondrial pathway (Yao et al., 2020). They also observed that GCs exposure resulted in mitochondrial fission and conspicuous abnormalities of ROS production and mitochondrial membrane potential (MMP), which promote the apoptosis of EPCs (Yao et al., 2020). Similarly, VO-OHpic could reverse these changes and protect EPCs. In addition, nuclear factor erythroid 2-related factor 2 (Nrf2) regulates the production of several antioxidant enzymes (Tavakkoli et al., 2019). VO-OHpic promotes angiogenesis and suppresses apoptosis of EPCs by activating Nrf2 (Yao et al., 2020). Therefore, VO-OHpic may be an effective strategy for the prevention and therapy of SONFH.

Bone tissue engineering is getting increasingly attractive to researchers because of the enormous potential for osteogenesis and angiogenesis. To enhance bone regeneration and angiogenesis at SONFH lesions, transplantation of BMSCs, EPCs and co-transplantation of both have been reported so far. BMSCs are considered to be ideal seed cells for SONFH treatment due to the enormous potential for self-renewal and multilineage differentiation, including osteogenesis and angiogenesis (Chen et al., 2019). However, several studies have reported that BMSCs isolated from proximal femur and iliac crest in SONFH patients have decreased osteogenic differentiation ability, limiting the application of BMSCs transplantation in SONFH treatment (Hernigou et al., 1999; Houdek et al., 2016). Although researchers have used gene transfection and established sustainable-release growth factor biomaterials to enhance BMSCs' osteogenic and angiogenic abilities, the harm of gene transfection to human body and the construction of suitable biomaterials remain incomplete problems (Wen et al., 2008b; Wen et al., 2012; Amsden, 2015; Shapiro et al., 2018; Kong et al., 2019). For EPCs, they are not directly involved in osteogenesis because they cannot differentiate into osteoblasts (Xu et al., 2021). Both carboxymethyl chitosan (CMC) and alginate (ALG) possess outstanding biocompatibility in enhancing osteogenesis (Upadhyaya et al., 2013; Jain and BarShalom, 2014). The composite scaffold can not only transport stem cells, but also provide a beneficial microenvironment for cell proliferation and intercellular communications (Xu et al., 2021). Therefore, CMC/ALG/BMSC/EPC composite scaffold have been developed for SONFH treatment and prevention.

Co-cultured BMECs and EPCs interact with each other through paracrine and direct intercellular contact to promote osteogenesis and angiogenesis has been verified as the main mechanism. Xu et al. demonstrated that BMSCs and EPCs mutually promote osteogenesis and angiogenesis through the secretion of various growth factors, such as VEGF and PDGF (Xu et al., 2021). Moreover, direct contact between EPCs and BMSCs can lead to endothelial-like phenotypic differentiation of BMSCs (Joddar et al., 2018). Implanted cells can promote tissue regeneration through proliferation, differentiation and paracrine (García-Sánchez et al., 2019). In addition to impaired osteogenesis and angiogenesis, lipid metabolism disturbance is another key promotor contributing to SONFH (Zhang et al., 2018). The imbalance between osteogenic and adipogenic differentiation of BMSCs may lead to adipocyte hypertrophy and fat embolism, reducing blood supply to the femoral head (Fukui et al., 2006). Transcription factors play a critical role in determining the fate of BMSCs. For instance, Runx2 and BMP-2 are crucial transcription factors that promote osteogenic differentiation of BMSCs, while $\operatorname{PPAR} \gamma$ and $\mathrm{C} / \mathrm{EBPa}$ are pivotal transcription factors that enhance adipogenic differentiation of BMSCs (Xu et al., 2021). The expression of Runx2 and BMP-2 was up-regulated in the co-cultured cells, while the expression of PPAR $\gamma$ and C/EBPa was down-regulated, resulting in BMSCs tending to differentiate into osteoblasts $\mathrm{Xu}$ 


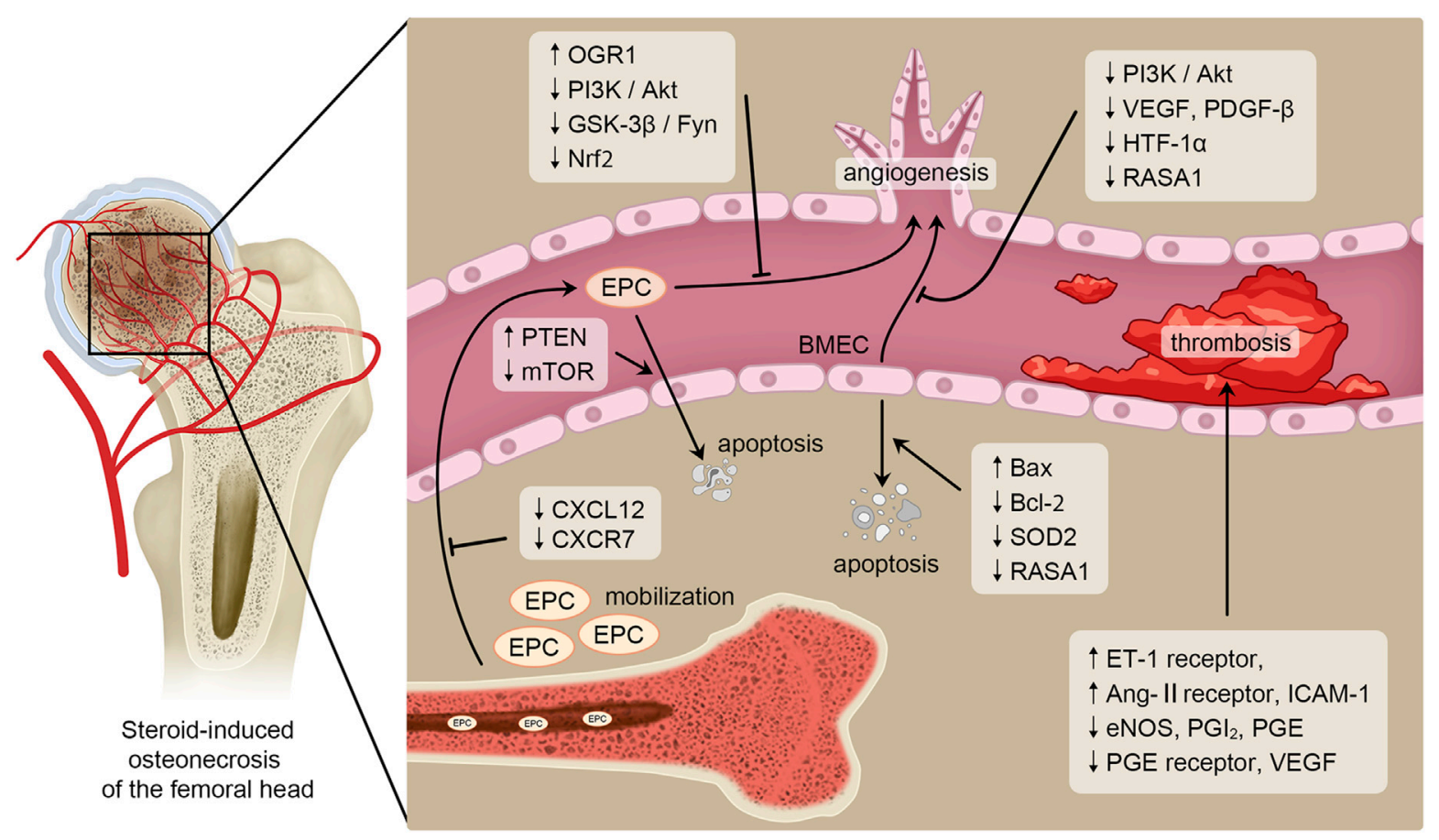

FIGURE 1 | Pathogenesis in SONFH related to bone endothelial cells. Blood vessels play a critical role in the occurrence and progression of SONFH. And bone endothelial cells are essential for maintaining vascular homeostasis and angiogenesis. Therefore, bone endothelial cells are key regulatory factors in the pathogenesis of SONFH. SONFH is affected by GCs regulating mobilization, angiogenesis, apoptosis and thrombosis of bone endothelial cells through several signaling pathways or cytokines such as PI3K/Akt, GSK-3ß/Fyn, Bcl-2 and Bax.

et al., 2021). However, the optimal ratio between BMSCs and EPCs in a co-transplantation system has yet to be determined. The SONFH lesion is in a state of hypoxia due to blood supply disorder, and the ability of proliferation, differentiation and cytokine secretion of co-cultured cells under hypoxia circumstances remains to be studied. In conclusion, BMSCs and EPCs co-transplantation is a promising therapeutic approach for SONFH.

\section{Human Experiments of Endothelial Progenitor Cells}

Endothelial Progenitor Cells used in human experiments were isolated and extracted from patients with SONFH. Feng et al. observed a decrease in the number and function of circulating EPCs in patients with SONFH, such as suppressed migration, impaired angiogenesis, and increased senescence (Feng et al., 2010). Similarly, Ding P. et al. (2019) reported that low doses of GCs significantly inhibited angiogenesis of EPCs, while only large doses of GCs could significantly inhibited cell proliferation. Clinical routine doses of GCs may never reach the threshold of serum concentration that inhibit EPCs proliferation (RousterStevens et al., 2008). And the decreased number of EPCs in patients with SONFH may as a result of the indirect effects of long-term exposure to GCs (Ding P. et al., 2019). In addition, GCs can down-regulate the expression of CXCR7 in EPCs and inhibit the downstream Akt and GSK-3 $\beta /$ Fyn signaling pathways of SDF-1/CXCR7 (Ding P. et al., 2019). Akt and GSK-3 $\beta /$ Fyn are involved in the angiogenesis of EPCs, and the up-regulation of Fyn caused by the decreased phosphorylation of GSK-3 $\beta$ can promote the degradation of Nrf2 (Torossian et al., 2014; Chen et al., 2015; Dai et al., 2017).

In addition, Chen et al. (2013) found that the migration and secretion of eEPCs were inhibited, while the proliferation and angiogenesis of lEPCs were significantly suppressed, which was appropriate for their different physiological functions. At the same time, the number of eEPCs and IEPCs were lower than that of the control group with similar conditions. Therefore, lEPCs may be a superior graft for SONFH compared to EPCs. Recent studies have reported successful enrichment and cultivation of IEPCs on a large scale, which greatly expanded the application prospect of IEPCs in bone tissue engineering (Reinisch et al., 2009; Kolbe et al., 2010).

In injured tissue, cells expressing CXCR4 are recruited through SDF-1 secreted by surrounding cells to promote healing of the injury (Ding and Tredget, 2015). Carolina et al. (2018) reported that GCs inhibited the migration and homing of umbilical cord blood (UCB) derived human EPCs to injury by down-regulating CXCR4 expression in both normoxic and hypoxic conditions. In normoxic conditions, GCs down-regulate CXCR4 expression in EPCs by damaging prostaglandin E2 (PGE2) synthases cyclooxygenase (COX2) and microsomal PGE2 synthase 1 (mPEGS1) and prostaglandin receptor EP4. While in hypoxic conditions, GCs down-regulate CXCR4 expression in EPCs through both PGE2 pathway and HIF2a pathway. However, whether GCs could influence the migration and homing ability 
TABLE 1 | Candidate reagents targeting bone endothelial cells to treat SONFH.

\begin{tabular}{|c|c|c|c|}
\hline $\begin{array}{l}\text { Candidate } \\
\text { reagents }\end{array}$ & Experimental models & Effects & References \\
\hline \multirow[t]{3}{*}{ ICA } & BMECs isolated from LPS and MPS treated rats & Decreased miR-335 to up-regulate the expression of eNOS, SOD2, RASA1 & $\begin{array}{l}\text { Yue et al. } \\
(2021)\end{array}$ \\
\hline & BMECs isolated from MPS treated rats & $\begin{array}{l}\text { Decreased the rate of empty lacunae and increased blood vessels and the } \\
\text { angiogenic biomarker CD31 }\end{array}$ & Yu et al. (2019) \\
\hline & $\begin{array}{l}\text { BMECs isolated from patients undergoing THA with } \\
\text { femoral neck fractures }\end{array}$ & $\begin{array}{l}\text { Promoted angiogenesis by up-regulating the expression of CD31, vWF, PDGF- } \\
\mathrm{B} \text { in BMECs and activating Akt and reduced the apoptosis of BMECs by up- } \\
\text { regulating Bax and down-regulating the expression of Bcl-2 }\end{array}$ & Yu et al. (2019) \\
\hline Pravastatin & EPCs isolated from Dex treated rats & $\begin{array}{l}\text { Activated AMPK mediated by LKB1, thereby inhibiting the mTOR signaling } \\
\text { pathway, recovering autophagy of EPCs and protecting them from Dex- } \\
\text { induced apoptosis }\end{array}$ & $\begin{array}{l}\text { Liao et al. } \\
(2018)\end{array}$ \\
\hline VO-OHpic & EPCs isolated from rats and treated with MPS & $\begin{array}{l}\text { Promoted angiogenesis and suppressed apoptosis through inhibiting } \\
\text { mitochondrial pathway and activating Nrf2 }\end{array}$ & $\begin{array}{l}\text { Yao et al. } \\
(2020)\end{array}$ \\
\hline
\end{tabular}

Abbreviations: ICA, icariin; BMECs, bone microvascular endothelial cells; LPS, lipopolysaccharide; MPS, methylprednisolone; eNOS, endothelial nitric oxide synthase; SOD2, superoxide dismutase 2; RASA1, Ras 21 protein activator 1; THA, total hip arthroplasty; VWF, von Willebrand factor; PDGF-B, platelet-derived growth factor-B; Bax, Bcl-2, associated X; BCl-2, B cell lymphoma-2; EPCs, endothelial progenitor cells; Dex, dexamethasone; AMPK, AMP-activated protein kinase; LKB1, liver kinase B1; mTOR, mammalian target of rapamycin; Nrf2, nuclear factor erythroid 2-related factor 2.

of bone marrow derived EPCs in SONFH patients remains to be further investigated.

\section{CONCLUSION}

SONFH is a disabling joint disease without effective drug treatment so far. Severe advanced SONFH can only be treated with THA, which may be accompanied by a series of side effects, including dislocation, periprosthetic fracture, infection and prosthesis loosening especially for young, active population. Impaired blood vessels is a key factor in many of the proposed pathogenesis of SONFH. Bone endothelial cells, including BMECs and their precursors, EPCs, both play a critical role in maintaining the normal structure and function of blood vessels. Impaired angiogenesis, abnormal apoptosis, thrombosis and fat embolism caused by the dysfunction of bone endothelial cells are involved in the occurrence and progression of SONFH (Figure 1). Therefore, ICA, pravastatin, and VO-OHpic are candidate reagents for the prevention and treatment of SONFH by promoting angiogenesis and inhibiting apoptosis and vascular embolization (Table 1). However, these reagents

\section{REFERENCES}

Ambros, V. (2004). The Functions of Animal microRNAs. Nature 431 (7006), 350-355. doi:10.1038/nature02871

Amsden, B. (2015). Novel Biodegradable Polymers for Local Growth Factor Delivery. Eur. J. Pharmaceutics Biopharmaceutics 97 (Pt B), 318-328. doi:10.1016/j.ejpb.2015.06.008

Asahara, T., Kawamoto, A., and Masuda, H. (2011). Concise Review: Circulating Endothelial Progenitor Cells for Vascular Medicine. Stem Cells 29 (11), 1650-1655. doi:10.1002/stem.745

Balistreri, C. R., Buffa, S., Pisano, C., Lio, D., Ruvolo, G., and Mazzesi, G. (2015). Are Endothelial Progenitor Cells the Real Solution for Cardiovascular Diseases? Focus on Controversies and Perspectives. Biomed. Res. Int. 2015, 1-17. doi: $10.1155 / 2015 / 835934$ are still in the preclinical stage and are not yet sufficient for widespread clinical use. In addition, bone tissue engineering such as bone endothelial cells and BMSCs co-transplantation is one of the most promising strategies for treating SONFH. The optimal ratio between cultured cells of co-grafts and scaffolds with excellent biocompatibility need further investigations.

\section{AUTHOR CONTRIBUTIONS}

$\mathrm{CH}$ and $\mathrm{ZW}$ performed the majority of the literature search and primarily drafted this manuscript. JN and SL assisted with the literature search. WW supervised the whole work. All authors read and approved the final manuscript.

\section{FUNDING}

This work was supported by grants (81902203 to $\mathrm{CH}$ and 81972107 to WW) from the National Natural Science Foundation of China and grant (ZRJY2021-QM21 to CH) from the Elite Medical Professionals Project of China-Japan Friendship Hospital.

Boyer, M., Townsend, L. E., Vogel, L. M., Falk, J., Reitz-Vick, D., Trevor, K. T., et al. (2000). Isolation of Endothelial Cells and Their Progenitor Cells from Human Peripheral Blood. J. Vasc. Surg. 31 (1 Pt 1), 181-189. doi:10.1016/s07415214(00)70080-2

Brandi, M. L., and Collin-Osdoby, P. (2006). Vascular Biology and the Skeleton. J. Bone Miner Res. 21 (2), 183-192. doi:10.1359/jbmr.050917

Bui, T. M., Wiesolek, H. L., and Sumagin, R. (2020). ICAM-1: A Master Regulator of Cellular Responses in Inflammation, Injury Resolution, and Tumorigenesis. J. Leukoc. Biol. 108 (3), 787-799. doi:10.1002/jlb.2mr0220-549r

Carolina, E., Kato, T., Khanh, V. C., Moriguchi, K., Yamashita, T., Takeuchi, K., et al. (2018). Glucocorticoid Impaired the Wound Healing Ability of Endothelial Progenitor Cells by Reducing the Expression of CXCR4 in the PGE2 Pathway. Front. Med. 5, 276. doi:10.3389/fmed.2018.00276

Chen, C., Yang, S., Feng, Y., Wu, X., Chen, D., Yu, Q., et al. (2013). Impairment of Two Types of Circulating Endothelial Progenitor Cells in Patients with 
Glucocorticoid-Induced Avascular Osteonecrosis of the Femoral Head. Jt. Bone Spine 80 (1), 70-76. doi:10.1016/j.jbspin.2012.02.015

Chen, D., Xia, Y., Zuo, K., Wang, Y., Zhang, S., Kuang, D., et al. (2015). Crosstalk between SDF-1/CXCR4 and SDF-1/CXCR7 in Cardiac Stem Cell Migration. Sci. Rep. 5, 16813. doi:10.1038/srep16813

Chen, X.-J., Shen, Y.-S., He, M.-C., Yang, F., Yang, P., Pang, F.-X., et al. (2019). Polydatin Promotes the Osteogenic Differentiation of Human Bone Mesenchymal Stem Cells by Activating the BMP2-Wnt/ $\beta$-Catenin Signaling Pathway. Biomed. Pharmacother. 112, 108746. doi:10.1016/ j.biopha.2019.108746

Dai, X., Yan, X., Zeng, J., Chen, J., Wang, Y., Chen, J., et al. (2017). Elevating CXCR7 Improves Angiogenic Function of EPCs via Akt/GSK-3 $\beta /$ FynMediated Nrf2 Activation in Diabetic Limb Ischemia. Circ. Res. 120 (5), e7-e23. doi:10.1161/circresaha.117.310619

Delbridge, A. R. D., Grabow, S., Strasser, A., and Vaux, D. L. (2016). Thirty Years of BCL-2: Translating Cell Death Discoveries into Novel Cancer Therapies. Nat. Rev. Cancer 16 (2), 99-109. doi:10.1038/nrc.2015.17

Dimova, I., Karthik, S., Makanya, A., Hlushchuk, R., Semela, D., Volarevic, V., et al. (2019). SDF-1/CXCR4 Signalling Is Involved in Blood Vessel Growth and Remodelling by Intussusception. J. Cel Mol Med 23 (6), 3916-3926. doi: $10.1111 /$ jcmm. 14269

Ding, J., and Tredget, E. E. (2015). The Role of Chemokines in Fibrotic Wound Healing. Adv. Wound Care 4 (11), 673-686. doi:10.1089/wound.2014.0550

Ding, P., Zhang, W., Tan, Q., Yao, C., and Lin, S. (2019a). Impairment of Circulating Endothelial Progenitor Cells (EPCs) in Patients with Glucocorticoid-Induced Avascular Necrosis of the Femoral Head and Changes of EPCs after Glucocorticoid Treatment In Vitro. J. Orthop. Surg. Res. 14 (1), 226. doi:10.1186/s13018-019-1279-6

Ding, S., Xu, J., Zhang, Q., Chen, F., Zhang, J., Gui, K., et al. (2019b). OGR1 Mediates the Inhibitory Effects of Acidic Environment on Proliferation and Angiogenesis of Endothelial Progenitor Cells. Cell Biol Int 43 (11), 1307-1316. doi:10.1002/cbin.11179

Doyle, B., and Caplice, N. (2005). A New Source of Endothelial Progenitor Cells Vascular Biology Redefined? Trends Biotechnol. 23 (9), 444-446. doi:10.1016/ j.tibtech.2005.05.013

Drescher, W., Pufe, T., Smeets, R., Eisenhart-Rothe, R. v., Jäger, M., and Tingart, M. (2011). Hüftkopfnekrose - Diagnostik und Differenzialtherapie. Z. Orthop. Unfall 149 (2), 231-242. doi:10.1055/s-0030-1270984

Eisenberg-Lerner, A., Bialik, S., Simon, H.-U., and Kimchi, A. (2009). Life and Death Partners: Apoptosis, Autophagy and the Cross-Talk between Them. Cell Death Differ 16 (7), 966-975. doi:10.1038/cdd.2009.33

Fang, W.-t., Li, H.-j., and Zhou, L.-s. (2010). Protective Effects of Prostaglandin E1 on Human Umbilical Vein Endothelial Cell Injury Induced by Hydrogen Peroxide. Acta Pharmacol. Sin 31 (4), 485-492. doi:10.1038/aps.2010.23

Feng, Y., Yang, S.-H., Xiao, B.-J., Xu, W.-H., Ye, S.-N., Xia, T., et al. (2010). Decreased in the Number and Function of Circulation Endothelial Progenitor Cells in Patients with Avascular Necrosis of the Femoral Head. Bone 46 (1), 32-40. doi:10.1016/j.bone.2009.09.001

Finney, M. R., Greco, N. J., Haynesworth, S. E., Martin, J. M., Hedrick, D. P., Swan, J. Z., et al. (2006). Direct Comparison of Umbilical Cord Blood versus Bone Marrow-Derived Endothelial Precursor Cells in Mediating Neovascularization in Response to Vascular Ischemia. Biol. Blood Marrow Transplant. 12 (5), 585-593. doi:10.1016/j.bbmt.2005.12.037

Fukui, K., Kominami, R., Shinohara, H., and Matsumoto, T. (2006). Glucocorticoid Induces Micro-fat Embolism in the Rabbit: a Scanning Electron Microscopic Study. J. Orthop. Res. 24 (4), 675-683. doi:10.1002/jor.20091

García-Sánchez, D., Fernández, D., Rodríguez-Rey, J. C., and Pérez-Campo, F. M. (2019). Enhancing Survival, Engraftment, and Osteogenic Potential of Mesenchymal Stem Cells. Wjsc 11 (10), 748-763. doi:10.4252/wjsc.v11.i10.748

Ghosh, A. K., and Vaughan, D. E. (2012). PAI-1 in Tissue Fibrosis. J. Cel. Physiol. 227 (2), 493-507. doi:10.1002/jcp.22783

Hamacher-Brady, A., Brady, N. R., and Gottlieb, R. A. (2006). Enhancing Macroautophagy Protects against Ischemia/reperfusion Injury in Cardiac Myocytes. J. Biol. Chem. 281 (40), 29776-29787. doi:10.1074/jbc.M603783200

Hernigou, P., Beaujean, F., and Lambotte, J. C. (1999). Decrease in the Mesenchymal Stem-Cell Pool in the Proximal Femur in CorticosteroidInduced Osteonecrosis. The J. Bone Jt. Surg. Br. volume 81-B (2), 349-355. doi:10.1302/0301-620x.81b2.881810.1302/0301-620x.81b2.0810349
Hirano, K., Tsutsui, H., Sugioka, Y., and Sueishi, K. (1997). Histopathologic Alterations of Retinacular Vessels and Osteonecrosis. Clin. Orthopaedics Relat. Res. 342, 192-204. doi:10.1097/00003086-199709000-00027

Hirschi, K. K., Ingram, D. A., and Yoder, M. C. (2008). Assessing Identity, Phenotype, and Fate of Endothelial Progenitor Cells. Atvb 28 (9), 1584-1595. doi:10.1161/atvbaha.107.155960

Hong, F.-f., Liang, X.-y., Liu, W., Lv, S., He, S.-j., Kuang, H.-b., et al. (2019). Roles of eNOS in Atherosclerosis Treatment. Inflamm. Res. 68 (6), 429-441. doi:10.1007/s00011-019-01229-9

Houde, M., Desbiens, L., and D’Orléans-Juste, P. (2016). Endothelin-1. Adv. Pharmacol. 77, 143-175. doi:10.1016/bs.apha.2016.05.002

Houdek, M. T., Wyles, C. C., Packard, B. D., Terzic, A., Behfar, A., and Sierra, R. J. (2016). Decreased Osteogenic Activity of Mesenchymal Stem Cells in Patients with Corticosteroid-Induced Osteonecrosis of the Femoral Head. The J. Arthroplasty 31 (4), 893-898. doi:10.1016/j.arth.2015.08.017

Ingram, D. A., Mead, L. E., Tanaka, H., Meade, V., Fenoglio, A., Mortell, K., et al. (2004). Identification of a Novel Hierarchy of Endothelial Progenitor Cells Using Human Peripheral and Umbilical Cord Blood. Blood 104 (9), 2752-2760. doi:10.1182/blood-2004-04-1396

Jain, D., and Bar-Shalom, D. (2014). Alginate Drug Delivery Systems: Application in Context of Pharmaceutical and Biomedical Research. Drug Development Ind. Pharm. 40 (12), 1576-1584. doi:10.3109/03639045.2014.917657

Joddar, B., Kumar, S. A., and Kumar, A. (2018). A Contact-Based Method for Differentiation of Human Mesenchymal Stem Cells into an Endothelial CellPhenotype. Cell Biochem Biophys 76 (1-2), 187-195. doi:10.1007/s12013-0170828-z

Johnson, A. J., Mont, M. A., Tsao, A. K., and Jones, L. C. (2014). Treatment of Femoral Head Osteonecrosis in the United States: 16-year Analysis of the Nationwide Inpatient Sample. Clin. Orthop. Relat. Res. 472 (2), 617-623. doi:10.1007/s11999-013-3220-3

Kerachian, M. A., Harvey, E. J., Cournoyer, D., Chow, T. Y. K., and Séguin, C. (2006). Avascular Necrosis of the Femoral Head: Vascular Hypotheses. Endothelium 13 (4), 237-244. doi:10.1080/10623320600904211

Kerachian, M. A., Séguin, C., and Harvey, E. J. (2009a). Glucocorticoids in Osteonecrosis of the Femoral Head: a New Understanding of the Mechanisms of Action. J. Steroid Biochem. Mol. Biol. 114 (3-5), 121-128. doi:10.1016/j.jsbmb.2009.02.007

Kim, S.-W., Kim, H., Cho, H.-J., Lee, J.-U., Levit, R., and Yoon, Y.-s. (2010). Human Peripheral Blood-Derived CD31+Cells Have Robust Angiogenic and Vasculogenic Properties and Are Effective for Treating Ischemic Vascular Disease. J. Am. Coll. Cardiol. 56 (7), 593-607. doi:10.1016/ j.jacc.2010.01.070

Kolbe, M., Dohle, E., Katerla, D., Kirkpatrick, C. J., and Fuchs, S. (2010). Enrichment of Outgrowth Endothelial Cells in High and Low colonyforming Cultures from Peripheral Blood Progenitors. Tissue Eng. C: Methods 16 (5), 877-886. doi:10.1089/ten.TEC.2009.0492

Kong, L., Zuo, R., Wang, M., Wang, W., Xu, J., Chai, Y., et al. (2020). Silencing MicroRNA-137-3p, Which Targets RUNX2 and CXCL12 Prevents SteroidInduced Osteonecrosis of the Femoral Head by Facilitating Osteogenesis and Angiogenesis. Int. J. Biol. Sci. 16 (4), 655-670. doi:10.7150/ijbs.38713

Kong, Y., Zhao, Y., Li, D., Shen, H., and Yan, M. (2019). Dual Delivery of Encapsulated BM-MSCs and BMP-2 Improves Osteogenic Differentiation and New Bone Formation. J. Biomed. Mater. Res. 107 (10), 2282-2295. doi:10.1002/jbm.a.36737

Kotelevets, L., Scott, M. G. H., and Chastre, E. (2018). Targeting PTEN in Colorectal Cancers. Adv. Exp. Med. Biol. 1110, 55-73. doi:10.1007/978-3030-02771-1_5

Krol, J., Loedige, I., and Filipowicz, W. (2010). The Widespread Regulation of microRNA Biogenesis, Function and Decay. Nat. Rev. Genet. 11 (9), 597-610. doi:10.1038/nrg2843

Kusumbe, A. P., Ramasamy, S. K., and Adams, R. H. (2014). Coupling of Angiogenesis and Osteogenesis by a Specific Vessel Subtype in Bone. Nature 507 (7492), 323-328. doi:10.1038/nature13145

Lan, H., Lu, H., Wang, X., and Jin, H. (2015). MicroRNAs as Potential Biomarkers in Cancer: Opportunities and Challenges. Biomed. Res. Int. 2015, 1-17. doi:10.1155/2015/125094

Lane, N. E., Mohan, G., Yao, W., Shidara, K., Lay, Y.-A. E., Junjing, J., et al. (2018). Prevalence of Glucocorticoid Induced Osteonecrosis in the Mouse Is Not 
Affected by Treatments that Maintain Bone Vascularity. Bone Rep. 9, 181-187. doi:10.1016/j.bonr.2018.10.003

Lee, M. Y., Luciano, A. K., Ackah, E., Rodriguez-Vita, J., Bancroft, T. A., Eichmann, A., et al. (2014). Endothelial Akt1 Mediates Angiogenesis by Phosphorylating Multiple Angiogenic Substrates. Proc. Natl. Acad. Sci. 111 (35), 12865-12870. doi:10.1073/pnas.1408472111

Lei, Z., van Mil, A., Brandt, M. M., Grundmann, S., Hoefer, I., Smits, M., et al. (2015). MicroRNA-132/212 Family Enhances Arteriogenesis after Hindlimb Ischaemia through Modulation of the Ras-MAPK Pathway. J. Cel. Mol. Med. 19 (8), 1994-2005. doi:10.1111/jcmm.12586

Li, T., Zhang, Y., Wang, R., Xue, Z., Li, S., Cao, Y., et al. (2019). Discovery and Validation an Eight-Biomarker Serum Gene Signature for the Diagnosis of Steroid-Induced Osteonecrosis of the Femoral Head. Bone 122, 199-208. doi:10.1016/j.bone.2019.03.008

Li, W., Zhang, T., Guo, L., and Huang, L. (2018). Regulation of PTEN Expression by Noncoding RNAs. J. Exp. Clin. Cancer Res. 37 (1), 223. doi:10.1186/s13046018-0898-9

Li, X., Jin, L., Cui, Q., Wang, G.-J., and Balian, G. (2005). Steroid Effects on Osteogenesis through Mesenchymal Cell Gene Expression. Osteoporos. Int. 16 (1), 101-108. doi:10.1007/s00198-004-1649-7

Li, Y., Chen, J., Zhang, Z., Wang, K., Tong, Z., and Yan, H. (2004). The Experimental Study on Treatment of Glucocorticoid-Induced Ischemic Necrosis of Femoral Head by Gu Fu Sheng Capsule. J. Tradit Chin. Med. 24 (4), 303-307.

Liao, Y., Su, R., Zhang, P., Yuan, B., and Li, L. (2017). Cortisol Inhibits mTOR Signaling in Avascular Necrosis of the Femoral Head. J. Orthop. Surg. Res. 12 (1), 154. doi:10.1186/s13018-017-0656-2

Liao, Y., Zhang, P., Yuan, B., Li, L., and Bao, S. (2018). Pravastatin Protects against Avascular Necrosis of Femoral Head via Autophagy. Front. Physiol. 9, 307. doi:10.3389/fphys.2018.00307

Lu, Y., Yu, Q., Guo, W., Hao, Y., Sun, W., and Cheng, L. (2020). Effect of Glucocorticoids on the Function of Microvascular Endothelial Cells in the Human Femoral Head Bone. Adv. Clin. Exp. Med. 29 (3), 345-353. doi:10.17219/acem/112602

Madonna, R., and De Caterina, R. (2015). Circulating Endothelial Progenitor Cells: Do They Live up to Their Name? Vasc. Pharmacol. 67-69, 2-5. doi:10.1016/ j.vph.2015.02.018

Mao, Z., Liu, G., Xiao, G. Y., Zhao, C., and Zou, Y. C. (2021). CircCDRlas Suppresses Bone Microvascular Endothelial Cell Activity and Angiogenesis through Targeting miR -135b/FIH -1 Axis. Orthop. Surg. 13 (2), 573-582. doi:10.1111/os.12883

Mead, L. E., Prater, D., Yoder, M. C., and Ingram, D. A. (2008). Isolation and Characterization of Endothelial Progenitor Cells from Human Blood. Curr. Protoc. Stem Cel Biol. 6, 1-26. doi:10.1002/9780470151808.sc02c01s6

Mittermayr, R., Slezak, P., Haffner, N., Smolen, D., Hartinger, J., Hofmann, A., et al. (2016). Controlled Release of Fibrin Matrix-Conjugated Platelet Derived Growth Factor Improves Ischemic Tissue Regeneration by Functional Angiogenesis. Acta Biomater. 29, 11-20. doi:10.1016/j.actbio.2015.10.028

Muraoka, N., Shum, L., Fukumoto, S., Nomura, T., Ohishi, M., and Nonaka, K. (2005). Transforming Growth Factor-B3 Promotes Mesenchymal Cell Proliferation and Angiogenesis Mediated by the Enhancement Ofcyclin D1,Flk-1, andCD31 Gene Expression during CL/Fr Mouse Lip Fusion. Birth Defect Res. A. 73 (12), 956-965. doi:10.1002/bdra.20191

Murata, M., Kumagai, K., Miyata, N., Osaki, M., and Shindo, H. (2007). Osteonecrosis in Stroke-Prone Spontaneously Hypertensive Rats: Effect of Glucocorticoid. J. Orthopaedic Sci. 12 (3), 289-295. doi:10.1007/s00776-007$1129-y$

Nguyen, N. H., Tran, G.-B., and Nguyen, C. T. (2020). Anti-oxidative Effects of Superoxide Dismutase 3 on Inflammatory Diseases. J. Mol. Med. 98 (1), 59-69. doi:10.1007/s00109-019-01845-2

Nishimura, T., Matsumoto, T., Nishino, M., and Tomita, K. (1997). Histopathologic Study of Veins in Steroid Treated Rabbits. Clin. Orthopaedics Relat. Res. 334, 37-42. doi:10.1097/00003086-199701000-00006

Nomura, M., Shimizu, S., Ito, T., Narita, M., Matsuda, H., and Tsujimoto, Y. (1999). Apoptotic Cytosol Facilitates Bax Translocation to Mitochondria that Involves Cytosolic Factor Regulated by Bcl-2. Cancer Res. 59 (21), 5542-5548.

Pacilli, A., and Pasquinelli, G. (2009). Vascular wall Resident Progenitor Cells. Exp. Cel Res. 315 (6), 901-914. doi:10.1016/j.yexcr.2008.12.018
Patel, J., Donovan, P., and Khosrotehrani, K. (2016). Concise Review: Functional Definition of Endothelial Progenitor Cells: A Molecular Perspective. Stem Cell Transl Med 5 (10), 1302-1306. doi:10.5966/sctm.2016-0066

Peng, Y., Wu, S., Li, Y., and Crane, J. L. (2020). Type H Blood Vessels in Bone Modeling and Remodeling. Theranostics 10 (1), 426-436. doi:10.7150/ thno.34126

Peters, E. B. (2018). Endothelial Progenitor Cells for the Vascularization of Engineered Tissues. Tissue Eng. B: Rev. 24 (1), 1-24. doi:10.1089/ ten.TEB.2017.0127

Phemister, D. B. (1935). BONE GROWTH AND REPAIR. Ann. Surg. 102 (2), 261-285. doi:10.1097/00000658-193508000-00013

Porter, A. G., and Jänicke, R. U. (1999). Emerging Roles of Caspase-3 in Apoptosis. Cel Death Differ 6 (2), 99-104. doi:10.1038/sj.cdd.4400476

Recchioni, R., Marcheselli, F., Antonicelli, R., Lazzarini, R., Mensà, E., Testa, R., et al. (2016). Physical Activity and Progenitor Cell-Mediated Endothelial Repair in Chronic Heart Failure: Is There a Role for Epigenetics? Mech. Ageing Development 159, 71-80. doi:10.1016/j.mad.2016.03.008

Rehman, J., Li, J., Orschell, C. M., and March, K. L. (2003). Peripheral Blood "endothelial Progenitor Cells" Are Derived from Monocyte/macrophages and Secrete Angiogenic Growth Factors. Circulation 107 (8), 1164-1169. doi:10.1161/01.cir.0000058702.69484.a0

Reinisch, A., Hofmann, N. A., Obenauf, A. C., Kashofer, K., Rohde, E., Schallmoser, K., et al. (2009). Humanized Large-Scale Expanded Endothelial colony-forming Cells Function In Vitro and In Vivo. Blood 113 (26), 6716-6725. doi:10.1182/ blood-2008-09-181362

Riddle, R. C., Khatri, R., Schipani, E., and Clemens, T. L. (2009). Role of HypoxiaInducible Factor-1 $\alpha$ in Angiogenic-Osteogenic Coupling. J. Mol. Med. 87 (6), 583-590. doi:10.1007/s00109-009-0477-9

Rouster-Stevens, K. A., Gursahaney, A., Ngai, K.-1., Daru, J. A., and Pachman, L. M. (2008). Pharmacokinetic Study of Oral Prednisolone Compared with Intravenous Methylprednisolone in Patients with Juvenile Dermatomyositis. Arthritis Rheum. 59 (2), 222-226. doi:10.1002/art.23341

Saito, S., Ohzono, K., and Ono, K. (1992). Early Arteriopathy and Postulated Pathogenesis of Osteonecrosis of the Femoral Head. Clin. Orthopaedics Relat. Res. \&NA; 98-110. doi:10.1097/00003086-199204000-00012

Shapiro, G., Lieber, R., Gazit, D., and Pelled, G. (2018). Recent Advances and Future of Gene Therapy for Bone Regeneration. Curr. Osteoporos. Rep. 16 (4), 504-511. doi:10.1007/s11914-018-0459-3

Shatanawi, A., Lemtalsi, T., Yao, L., Patel, C., Caldwell, R. B., and Caldwell, R. W. (2015). Angiotensin II Limits NO Production by Upregulating Arginase through a P38 MAPK-ATF-2 Pathway. Eur. J. Pharmacol. 746, 106-114. doi:10.1016/j.ejphar.2014.10.042

Song, H.-J., Lan, B. S., Cheng, B., Zhang, K. F., Yan, H. W., Wang, W. Z., et al. (2010). Peripheral Blood Stem Cell Transplantation for Ischemic Femoral Head Necrosis. Transplant. Proc. 42 (5), 1862-1864. doi:10.1016/ j.transproceed.2010.02.077

Starklint, H., Lausten, G. S., and Arnoldi, C. C. (1995). Microvascular Obstruction in Avascular Necrosis Immunohistochemistry of 14 Femoral Heads. Acta Orthopaedica Scand. 66 (1), 9-12. doi:10.3109/17453679508994629

Sun, Z.-B., Wang, J.-W., Xiao, H., Zhang, Q.-S., Kan, W.-S., Mo, F.-B., et al. (2015). Icariin May Benefit the Mesenchymal Stem Cells of Patients with Steroid-Associated Osteonecrosis by ABCB1-Promoter Demethylation: a Preliminary Study. Osteoporos. Int. 26 (1), 187-197. doi:10.1007/s00198014-2809-z

Tavakkoli, A., Iranshahi, M., Hasheminezhad, S. H., Hayes, A. W., and Karimi, G. (2019). The Neuroprotective Activities of Natural Products through the Nrf2 Upregulation. Phytotherapy Res. 33 (9), 2256-2273. doi:10.1002/ptr.6427

Thorburn, A. (2004). Death Receptor-Induced Cell Killing. Cell Signal. 16 (2), 139-144. doi:10.1016/j.cellsig.2003.08.007

Torossian, F., Anginot, A., Chabanon, A., Clay, D., Guerton, B., Desterke, C., et al. (2014). CXCR7 Participates in CXCL12-Induced CD34+ Cell Cycling through $\beta$-arrestin-dependent Akt Activation. Blood 123 (2), 191-202. doi:10.1182/ blood-2013-05-500496

Upadhyaya, L., Singh, J., Agarwal, V., and Tewari, R. P. (2013). Biomedical Applications of Carboxymethyl Chitosans. Carbohydr. Polym. 91 (1), 452-466. doi:10.1016/j.carbpol.2012.07.076

Uras, N., Oguz, S. S., Zergeroglu, S., Akdag, A., Polat, B., Dizdar, E. A., et al. (2012). CD31 and Factor VIII in Angiogenesis of normal and Pre-eclamptic Human 
Placentas. J. Obstet. Gynaecol. 32 (6), 533-536. doi:10.3109/ 01443615.2012.677875

Vogt, C., and Schmid-Schönbein, G. (2001). Microvascular Endothelial Cell Death and Rarefaction in the Glucocorticoid-Induced Hypertensive Rat. Umic 8 (2), 129-139. doi:10.1080/713774024

Wen, Q., Jin, D., Zhou, C.-Y., Zhou, M.-Q., Luo, W., and Ma, L. (2012). HGFtransgenic MSCs Can Improve the Effects of Tissue Self-Repair in a Rabbit Model of Traumatic Osteonecrosis of the Femoral Head. PLoS One 7 (5), e37503. doi:10.1371/journal.pone. 0037503

Wen, Q., Ma, L., Chen, Y.-P., Yang, L., Luo, W., and Wang, X.-N. (2008a). A Rabbit Model of Hormone-Induced Early Avascular Necrosis of the Femoral Head. Biomed. Environ. Sci. 21 (5), 398-403. doi:10.1016/ s0895-3988(08)60060-4

Wen, Q., Ma, L., Chen, Y.-P., Yang, L., Luo, W., and Wang, X.-N. (2008b). Treatment of Avascular Necrosis of the Femoral Head by Hepatocyte Growth Factor-Transgenic Bone Marrow Stromal Stem Cells. Gene Ther. 15 (23), 1523-1535. doi:10.1038/gt.2008.110

Werner, N., and Nickenig, G. (2006). Clinical and Therapeutical Implications of EPC Biology in Atherosclerosis. J. Cel Mol Med 10 (2), 318-332. doi:10.1111/ j.1582-4934.2006.tb00402.x

Wu, H., Riha, G. M., Yang, H., Li, M., Yao, Q., and Chen, C. (2005). Differentiation and Proliferation of Endothelial Progenitor Cells from Canine Peripheral Blood Mononuclear Cells1,2. J. Surg. Res. 126 (2), 193-198. doi:10.1016/ j.jss.2005.01.016

Wu, J., Qi, X., Liu, L., Hu, X., Liu, J., Yang, J., et al. (2019). Emerging Epigenetic Regulation of Circular RNAs in Human Cancer. Mol. Ther. - Nucleic Acids 16, 589-596. doi:10.1016/j.omtn.2019.04.011

Xu, H., Wang, C., Liu, C., Peng, Z., Li, J., Jin, Y., et al. (2021). Cotransplantation of Mesenchymal Stem Cells and Endothelial Progenitor Cells for Treating Steroidinduced Osteonecrosis of the Femoral Head. Stem Cell Transl Med 10 (5), 781-796. doi:10.1002/sctm.20-0346

Xu, R., Yallowitz, A., Qin, A., Wu, Z., Shin, D. Y., Kim, J.-M., et al. (2018). Targeting Skeletal Endothelium to Ameliorate Bone Loss. Nat. Med. 24 (6), 823-833. doi:10.1038/s41591-018-0020-z

Yang, C., Yang, S., Du, J., Li, J., Xu, W., and Xiong, Y. (2003). Vascular Endothelial Growth Factor Gene Transfection to Enhance the Repair of Avascular Necrosis of the Femoral Head of Rabbit. Chin. Med. J. (Engl) 116 (10), 1544-1548.

Yang, J.-X., Pan, Y.-Y., Wang, X.-X., Qiu, Y.-G., and Mao, W. (2018). Endothelial Progenitor Cells in Age-Related Vascular Remodeling. Cel Transpl. 27 (5), 786-795. doi:10.1177/0963689718779345

Yao, X., Yu, S., Jing, X., Guo, J., Sun, K., Guo, F., et al. (2020). PTEN Inhibitor VOOHpic Attenuates GC-Associated Endothelial Progenitor Cell Dysfunction and Osteonecrosis of the Femoral Head via Activating Nrf2 Signaling and Inhibiting Mitochondrial Apoptosis Pathway. Stem Cel Res Ther 11 (1), 140. doi:10.1186/ s13287-020-01658-y

Yavuz, B. B., Yavuz, B., Sener, D. D., Cankurtaran, M., Halil, M., Ulger, Z., et al. (2008). Advanced Age Is Associated with Endothelial Dysfunction in Healthy Elderly Subjects. Gerontology 54 (3), 153-156. doi:10.1159/000129064

Yeh, C.-H., Chang, J.-K., Wang, Y.-H., Ho, M.-L., and Wang, G.-J. (2008). Ethanol May Suppress Wnt/ $\beta$-Catenin Signaling on Human Bone Marrow Stroma Cells. Clin. Orthop. Relat. Res. 466 (5), 1047-1053. doi:10.1007/s11999-008-0171-1

Yu, H., Liu, P., Zuo, W., Sun, X., Liu, H., Lu, F., et al. (2020). Decreased Angiogenic and Increased Apoptotic Activities of Bone Microvascular Endothelial Cells in Patients with Glucocorticoid-Induced Osteonecrosis of the Femoral Head. BMC Musculoskelet. Disord. 21 (1), 277. doi:10.1186/s12891-020-03225-1

Yu, H., VandeVord, P. J., Mao, L., Matthew, H. W., Wooley, P. H., and Yang, S.-Y. (2009). Improved Tissue-Engineered Bone Regeneration by Endothelial Cell Mediated Vascularization. Biomaterials 30 (4), 508-517. doi:10.1016/ j.biomaterials.2008.09.047

Yu, H., Yue, J. a., Wang, W., Liu, P., Zuo, W., Guo, W., et al. (2019). Icariin Promotes Angiogenesis in Glucocorticoid-induced Osteonecrosis of Femoral
Heads: In Vitro and In Vivo Studies. J. Cel Mol Med 23 (11), 7320-7330. doi:10.1111/jcmm.14589

Yue, J. a., Wan, F., Zhang, Q., Wen, P., Cheng, L., Li, P., et al. (2018). Effect of Glucocorticoids on miRNA Expression Spectrum of Rat Femoral Head Microcirculation Endothelial Cells. Gene 651, 126-133. doi:10.1016/ j.gene.2018.01.057

Yue, J. a., Yu, H., Liu, P., Wen, P., Zhang, H., Guo, W., et al. (2021). Preliminary Study of Icariin Indicating Prevention of Steroid-Induced Osteonecrosis of Femoral Head by Regulating Abnormal Expression of miRNA-335 and Protecting the Functions of Bone Microvascular Endothelial Cells in Rats. Gene 766, 145128. doi:10.1016/j.gene.2020.145128

Zhang, G., Qin, L., and Shi, Y. (2007). Epimedium-derived Phytoestrogen Flavonoids Exert Beneficial Effect on Preventing Bone Loss in Late Postmenopausal Women: a 24-month Randomized, Double-Blind and Placebo-Controlled Trial. J. Bone Miner Res. 22 (7), 1072-1079. doi:10.1359/ jbmr.070405

Zhang, H., Wang, P., Zhang, X., Zhao, W., Ren, H., and Hu, Z. (2019). SDF1/ CXCR7 Signaling Axis Participates in Angiogenesis in Degenerated Discs via the PI3K/AKT Pathway. DNA Cel Biol. 38 (5), 457-467. doi:10.1089/ dna.2018.4531

Zhang, J., Pan, J., and Jing, W. (2020a). Motivating Role of Type H Vessels in Bone Regeneration. Cell Prolif 53 (9), e12874. doi:10.1111/cpr.12874

Zhang, Q., and L v, J. (2018). Role of Coagulopathy in Glucocorticoid-Induced Osteonecrosis of the Femoral Head. J. Int. Med. Res. 46 (6), 2141-2148. doi:10.1177/0300060517700299

Zhang, Y., Li, Y., Wang, Q., Su, B., Xu, H., Sun, Y., et al. (2020b). Role of RASA1 in Cancer: A Review and Update (Review). Oncol. Rep. 44 (6), 2386-2396. doi:10.3892/or.2020.7807

Zhao, D., Zhang, F., Wang, B., Liu, B., Li, L., Kim, S.-Y., et al. (2020). Guidelines for Clinical Diagnosis and Treatment of Osteonecrosis of the Femoral Head in Adults (2019 Version). J. Orthopaedic Translation 21, 100-110. doi:10.1016/ j.jot.2019.12.004

Zhou, C.-h., Meng, J.-h., Zhao, C.-c., Ye, C.-y., Zhu, H.-x., Hu, B., et al. (2017). PTH [1-34] Improves the Effects of Core Decompression in Early-Stage SteroidAssociated Osteonecrosis Model by Enhancing Bone Repair and Revascularization. PLoS One 12 (5), e0178781. doi:10.1371/ journal.pone. 0178781

Zhou, H., Mehta, S., Srivastava, S. P., Grabinska, K., Zhang, X., Wong, C., et al. (2020). Endothelial Cell-Glucocorticoid Receptor Interactions and Regulation of Wnt Signaling. JCI Insight 5 (3), el31384. doi:10.1172/jci.insight.131384

Zhu, H. M., Qin, L., Garnero, P., Genant, H. K., Zhang, G., Dai, K., et al. (2012). The First Multicenter and Randomized Clinical Trial of Herbal Fufang for Treatment of Postmenopausal Osteoporosis. Osteoporos. Int. 23 (4), 1317-1327. doi:10.1007/s00198-011-1577-2

Conflict of Interest: The authors declare that the research was conducted in the absence of any commercial or financial relationships that could be construed as a potential conflict of interest.

Publisher's Note: All claims expressed in this article are solely those of the authors and do not necessarily represent those of their affiliated organizations, or those of the publisher, the editors and the reviewers. Any product that may be evaluated in this article, or claim that may be made by its manufacturer, is not guaranteed or endorsed by the publisher.

Copyright $\odot 2021$ Huang, Wen, Niu, Lin and Wang. This is an open-access article distributed under the terms of the Creative Commons Attribution License (CC BY). The use, distribution or reproduction in other forums is permitted, provided the original author(s) and the copyright owner(s) are credited and that the original publication in this journal is cited, in accordance with accepted academic practice. No use, distribution or reproduction is permitted which does not comply with these terms. 